\title{
Effects of a-mangostin on embryonic development and liver development in zebrafish
}

\author{
Wittaya Pimtong ${ }^{1} \cdot$ Wannakarn Kitipaspallop ${ }^{2} \cdot$ Hang-Suk Chun ${ }^{3} \cdot$ Woo-Keun Kim ${ }^{3}$
}

Accepted: 31 August 2020 / Published online: 11 September 2020

(C) The Author(s) 2020

\begin{abstract}
Background Alpha-mangostin has potential as a chemopreventive agent but there is little information on its toxicological profile and developmental toxicity.

Objective We evaluated the effects of $\alpha$-mangostin on embryonic development and hepatogenesis in zebrafish.

Result Exposure of embryos to $0.25-4 \mu \mathrm{M} \alpha$-mangostin from 4-120 h post-fertilization (hpf) caused mortality of embryos with $\mathrm{LC}_{50} 1.48 \pm 0.29 \mu \mathrm{M}$. The compound also caused deformities, including head malformation, pericardial oedema, absence of swim bladder, yolk oedema, and bent tail. Exposure of zebrafish embryos to $\alpha$-mangostin during early hepatogenesis (16-72 hpf) decreased the transcript expression levels of liver fatty acid-binding protein 10a (Fabp10a), but increased gene markers of inflammation, oxidative stress, and apoptosis. In Fabp10a:DsRed transgenic zebrafish, the intensity and the area of fluorescence in the liver of the treated group were decreased (non-significantly) relative to controls.

Conclusion These effects were more marked during early hepatogenesis (16-72 hpf) than during post-hepatogenesis (72-120 hpf).
\end{abstract}

Keywords $\alpha$-Mangostin $\cdot$ Xanthone $\cdot$ Zebrafish $\cdot$ Hepatogenesis $\cdot$ Toxicity

\section{Introduction}

Claims for human health improvement have popularized mangosteen-based drinks. The mangosteen (Garcinia mangostana), a tropical fruit that mainly occurs in Southeast Asia, is used as a traditional medicine in the treatment of abdominal pain, infected wounds, and dysentery (PedrazaChaverri et al. 2008). Alpha-mangostin is one of the active compounds obtained from mangosteens (Jindarat 2014).

Electronic supplementary material The online version of this article (https://doi.org/10.1007/s13273-020-00099-1) contains supplementary material, which is available to authorized users.

Woo-Keun Kim

wookkim@kitox.re.kr

1 Nano Environmental and Health Safety Research Team, National Nanotechnology Center, National Science and Technology Development Agency, Khlong Luang, Pathum Thani 12120, Thailand

2 Department of Biology, Faculty of Science, Chulalongkorn University, Bangkok 10330, Thailand

3 Biosystem Research Group, Korea Institute of Toxicology, Daejeon 305-343, Republic of Korea
This compound has been investigated for biological properties, including antioxidant, antibacterial, anti-inflammatory, and anticancer activities (Pimtong et al. 2014; Wang et al. 2017; Chen et al. 2018). Several studies have shown $\alpha$-mangostin to be a promising candidate as an anticancer drug (Gavrilas et al. 2016; Hientz et al. 2017; Zhang et al. 2017). Alpha-mangostin is currently at the preclinical stage, where its toxicology, pharmacodynamics, and pharmacokinetics are extensively examined before clinical trials. However, to date, few studies have evaluated the toxicity of $\alpha$-mangostin.

After 48-h oral administration of $\alpha$-mangostin to rats with doses up to $1250 \mathrm{mg} / \mathrm{kg}$, the treated rats in both studies exhibited no toxic symptoms or mortality during the study period (Nelli et al. 2013; Kumar et al. 2016). In contrast, in a mouse model, Choi et al. found that $\alpha$-mangostin induced mortality after $72 \mathrm{~h}$ of intraperitoneal administration with $50 \%$ lethal concentration $\left(\mathrm{LC}_{50}\right)$ of $150 \mathrm{mg} / \mathrm{kg}$ (Choi et al. 2014). Furthermore, inclusion of $\alpha$-mangostin in mice diet at $250 \mathrm{mg} / \mathrm{kg}$ for 25 days caused intestinal dysbiosis (Han et al. 2015). Different sensitivities of these species might explain the difference between the results of the rat and mouse models. 
The thalidomide tragedy emphasized that obtaining safety information solely from rodent models is inadequate for human use of drugs. Thalidomide prescribed to pregnant women led to severe shortening, or complete absence, of limbs of babies. Subsequently, researchers found that thalidomide induces morphological abnormalities in other animal models, including monkey, rabbit, chick, and zebrafish (Ito et al. 2011; Vargesson 2015). Therefore, the toxicological profile of $\alpha$-mangostin in rodent models is insufficient for development of the drug to the clinical trial stage and safety information from other animal models is required. Furthermore, the effects of $\alpha$-mangostin on liver development have not been reported.

Zebrafish provide an alternative model that has been widely used in toxicological research. Zebrafish have many advantages, including possession of transparent embryos, rapid development, developmental pattern similar to that of mammals, and high genetic similarity to humans (Santoriello and Zon 2012). Some of their vital organs resemble those of mammals, including the liver. Hepatocyte precursors occur in zebrafish embryos during somite development at $16 \mathrm{~h}$ post-fertilization (hpf) (Korzh et al. 2001). By 22 $\mathrm{hpf}$, anterior endodermal progenitor cells gradually form the liver (Field et al. 2003). Between 24 and 28 hpf, hepatocyte aggregation eventually leads to looping of the intestinal primordium. By $50 \mathrm{hpf}$, the liver primordium is easily distinguished and then a period of hepatic outgrowth begins between 60-72 hpf. By the end of the outgrowth at $120 \mathrm{hpf}$, the liver consists of a large left lobe and a smaller right lobe, and is fully functional (Pack et al. 1996). Although zebrafish liver development is much more rapid than in rodent models, early hepatogenesis is similar to that of mice (Duncan 2013; Field et al. 2003; Ober et al. 2003).

Most drugs undergo chemical alteration, primarily in the liver, to create compounds more easily excreted from the body. Following intravenous and oral administration to mice, $\alpha$-mangostin was extensively metabolized in the liver (Han et al. 2015). Other studies have reported the therapeutic properties of $\alpha$-mangostin for the liver. Supawadee et al. (2015) showed that $\alpha$-mangostin reduced the risk of liver fibrosis in thioacetamide-induced liver cirrhosis in rats. The compound also inhibits in vitro hepatic stellate cell proliferation (Rahmaniah and Yuyuntia 2018). These studies suggest that $\alpha$-mangostin might decrease liver fibrosis in response to liver damage more generally. Furthermore, Kim et al. have reported that $\alpha$-mangostin ameliorates hepatic steatosis in high-fat diet-induced obese mice (Kim et al. 2017).

There are no reports on the effect of $\alpha$-mangostin on the liver or liver development. This study aimed to study the acute toxicity of $\alpha$-mangostin, its specific toxicity for hepatogenesis, and the mechanisms of $\alpha$-mangostininduced hepatotoxicity. The levels of genes involved in the fabp10a signalling pathway, oxidative stress, inflammation, and apoptosis were determined. A transgenic line $\operatorname{Tg}$ (fabp10a:DsRed) was also used as a model to study liver morphology.

\section{Materials and methods}

\section{Zebrafish maintenance}

Zebrafish wild-type $\mathrm{AB}$ line and the $\mathrm{Tg}$ (fabp10a:DsRed) transgenic line were raised and maintained in a temperaturecontrolled room $\left(28.5 \pm 1{ }^{\circ} \mathrm{C}\right)$ under a $14: 10 \mathrm{~h}$ light:dark photoperiod at the Korea Institute of Toxicology (KIT). All embryos were obtained from natural pairwise mating of adult zebrafish. The embryos were collected and selected under a stereomicroscope (SMZ645, Nikon Corp., Tokyo, Japan). All experimental procedures were approved by the KIT Institutional Animal Care and Use Committee (KIT-1907-0263).

\section{Zebrafish embryo acute toxicity testing of a-mangostin}

The embryo toxicity test was performed and evaluated according to the Organization for Economic Co-operation and Development for the testing of chemicals, No. 236 (OECD 2013). Fertilized eggs from wild-type zebrafish within 4 hpf were collected under a stereomicroscope (SZX7, Olympus Corp., Tokyo, Japan). Zebrafish embryos were transferred to a 12-well plate, 20 embryos/well in $2 \mathrm{~mL}$ of each concentration. The stock solution of $\alpha$-mangostin (Sigma-Aldrich Corp., St. Louis, MO) in DMSO (SigmaAldrich) was diluted to a range of concentrations: $0.25,0.5$, 1,2 , and $4 \mu \mathrm{M}$ in egg water $(60 \mu \mathrm{g} / \mathrm{mL}$ in Instant Ocean sea salt). The control vehicle was $0.4 \%$ DMSO. Each test solution was vortexed for $20 \mathrm{~s}$ before adding to the plates, which were then incubated at $28.5 \pm 1{ }^{\circ} \mathrm{C}$ for $120 \mathrm{~h}$. The test solutions were refreshed every $24 \mathrm{~h}$, and dead embryos were removed. Numbers of dead and abnormal embryos were recorded. The data were obtained from three independent experiments. Only experiments with $\geq 90 \%$ control survival were used in the analysis. The $\mathrm{LC}_{50}$ of $\alpha$-mangostin was derived by sigmoidal regression using SigmaPlot version 13 (Systat Software Inc., San Jose, CA).

\section{Testing of toxicological effects of a-mangostin on zebrafish hepatogenesis}

To study the effects of $\alpha$-mangostin on early hepatogenesis, $\operatorname{Tg}$ (fabp10a:DsRed) transgenic zebrafish embryos were used for two exposure periods: 16-72 hpf and 72-120 hpf. This transgenic line provides a useful tool in analyses of hepatotoxicity, indicated by changes of liver fluorescent 
intensity (Zhang et al. 2014). The transgenic model was used to neglect the breeding background between wild type and transgenic embryos, because we needed to use the transgenic model to study the effects of $\alpha$-mangostin on the liver phenotype subsequently. For the exposure, the embryos were transferred to a 24 -well plate at 10 embryos/well and exposed to $2 \mathrm{~mL}$ of $\alpha$-mangostin at concentrations of 0.5 , $1,2,4$ and $8 \mu \mathrm{m}$ at $28.5 \pm 1{ }^{\circ} \mathrm{C}$ until the endpoint. Zebrafish embryos treated with $0.4 \%$ DMSO were used as a vehicle control group. Triplicates for each concentration group were performed. Numbers of dead embryos and abnormal embryos were recorded. At the endpoint, zebrafish larvae were documented using a fluorescence stereomicroscope (M205 FA, Leica, Heerbrugg, Switzerland) equipped with a Leica DFC7000 T camera and Leica Application Suite X software. The fluorescence intensity and area were analysed and quantified using ImageJ software (available online: https ://imagej.nih.gov/ij/).

\section{Gene expression analysis by quantitative real-time PCR}

To gain more information on the effects of $\alpha$-mangostin on hepatogenesis, zebrafish larvae treated with $\alpha$-mangostin during hepatogenesis were analysed by quantitative realtime PCR (qRT-PCR). Total RNA was extracted from 40 homogenized larvae using the RNeasy mini kit (QIAGEN, Hilden, Germany) according to the manufacturer's instructions. The total RNA was then converted to cDNA using the iScript cDNA synthesis kit (Bio-Rad, Hercules, CA). Real-time PCR was performed in an Applied Biosystems StepOnePlus real-time PCR system (Applied Biosystems, Waltham, MA) using Power SYBR ${ }^{\circledR}$ Green PCR Master Mix (Applied Biosystems). Real-time PCR reaction was carried out with the following conditions profile: $2 \mathrm{~min}$ at $50{ }^{\circ} \mathrm{C}$, $10 \mathrm{~min}$ at $95^{\circ} \mathrm{C}$, followed by 40 cycles of $15 \mathrm{~s}$ at $95^{\circ} \mathrm{C}, 30 \mathrm{~s}$ at $60^{\circ} \mathrm{C}$, and $30 \mathrm{~s}$ at $72{ }^{\circ} \mathrm{C}$. A melting curve for each reaction was performed immediately after the amplification protocol with increments of $0.3{ }^{\circ} \mathrm{C}$ from 65 to $95{ }^{\circ} \mathrm{C}$. The primers were synthesized by Macrogen (Seoul, Republic of Korea) and their sequences were obtained from previous studies, as listed in Table S1. Gene expression changes were normalized to $\beta$-actin. All assays were performed in triplicate.

\section{Statistical analysis}

Data from individual assays were expressed as mean values \pm standard error (SE). Statistically significant differences among experimental groups were analysed by oneway analysis of variance (ANOVA) followed by post-hoc comparisons using Tukey's HSD tests (SPSS v20, IBM Corp., Armonk, NY). Differences among the groups were considered significant, where $p<0.05$.

\section{Results and discussion}

Several studies have investigated the pharmacological properties of $\alpha$-mangostin and have suggested that the compound has promising potential as an anticancer drug (Brito et al. 2017; Zhang et al. 2017). However, relatively few studies have evaluated the toxicological profile of $\alpha$-mangostin, and most of these have focused on rodent models (Her et al. 2003). An insufficiency of data on the safety profile of $\alpha$-mangostin impedes development of this drug to the clinical trial stage. Therefore, this study evaluated the toxicological effects of $\alpha$-mangostin on embryonic development and hepatogenesis in zebrafish.

\section{In acute toxicity tests a-mangostin causes mortality and malformations of zebrafish larvae}

To evaluate the toxicological effects of $\alpha$-mangostin on zebrafish embryonic development, zebrafish embryos at $4 \mathrm{hpf}$ were exposed to $\alpha$-mangostin $(0,0.25,0.5,1.0,2.0$, and $4.0 \mu \mathrm{M}$ ) until $120 \mathrm{hpf}$. Their survival rates are shown in Fig. 1. Alpha-mangostin induced mortality in was both concentration-dependent and time-dependent. Concentrations $<1 \mu \mathrm{M}$ had no significant effect on mortality by the end of the exposure. Exposure to $2 \mu \mathrm{M} \alpha$-mangostin induced about $80 \%$ mortality and exposure to $4 \mu \mathrm{M}$ $\alpha$-mangostin caused the death of all larvae. At the end of the exposure period, the derived $\mathrm{LC}_{50}$ was $1.48 \pm 0.29 \mu \mathrm{M}$. Alpha-mangostin did not affect the hatching rate of zebrafish embryos (data not shown). In our previous work, we evaluated the effects of $0-9 \mu \mathrm{M} \alpha$-mangostin during early development of zebrafish embryos (up to $72 \mathrm{hpf}$ ) (Kittipaspallop et al. 2019). Alpha-mangostin-induced mortality with $\mathrm{LC}_{50} 5.75 \pm 0.26 \mu \mathrm{M}$ but, similarly, we observed no effect on hatching rate. To eliminate the possibility of $\mathrm{pH}$-induced effects in the present work, the $\mathrm{pH}$ of the test solutions was measured; $\alpha$-mangostin did not change the $\mathrm{pH}$ of the embryonic medium (data not shown).

Phenotypic defects caused by $\alpha$-mangostin were examined at $120 \mathrm{hpf}$. No malformation were observed in groups with $\alpha$-mangostin concentrations $<0.5 \mu \mathrm{M}$ (Fig. 2a). At $1 \mu \mathrm{M}$, there were a few malformed embryos, but the number was not significantly different from that of the control group (Chi-squared test). At $2 \mu \mathrm{M}$, all surviving larvae exhibited deformities, including head malformation, pericardial oedema, absence of swim bladder, yolk oedema, and bent tail (Fig. 2b). Similar defects in zebrafish larvae were reported in our previous studies with 72-h exposure (Kittipaspallop et al. 2019). The present study is the first to report the acute toxicity of $\alpha$-mangostin in zebrafish embryos with exposures up to $120 \mathrm{hpf}$. 
Fig. 1 Cumulative survival rate of zebrafish larvae after exposure to $\alpha$-mangostin at various concentrations from $4 \mathrm{hpf}$ up to $120 \mathrm{hpf}$. Data are presented as means \pm SE of three independent experiments. Significant differences were analysed using one-way ANOVA followed by Tukey's HSD test. *** $p<0.001$ versus control

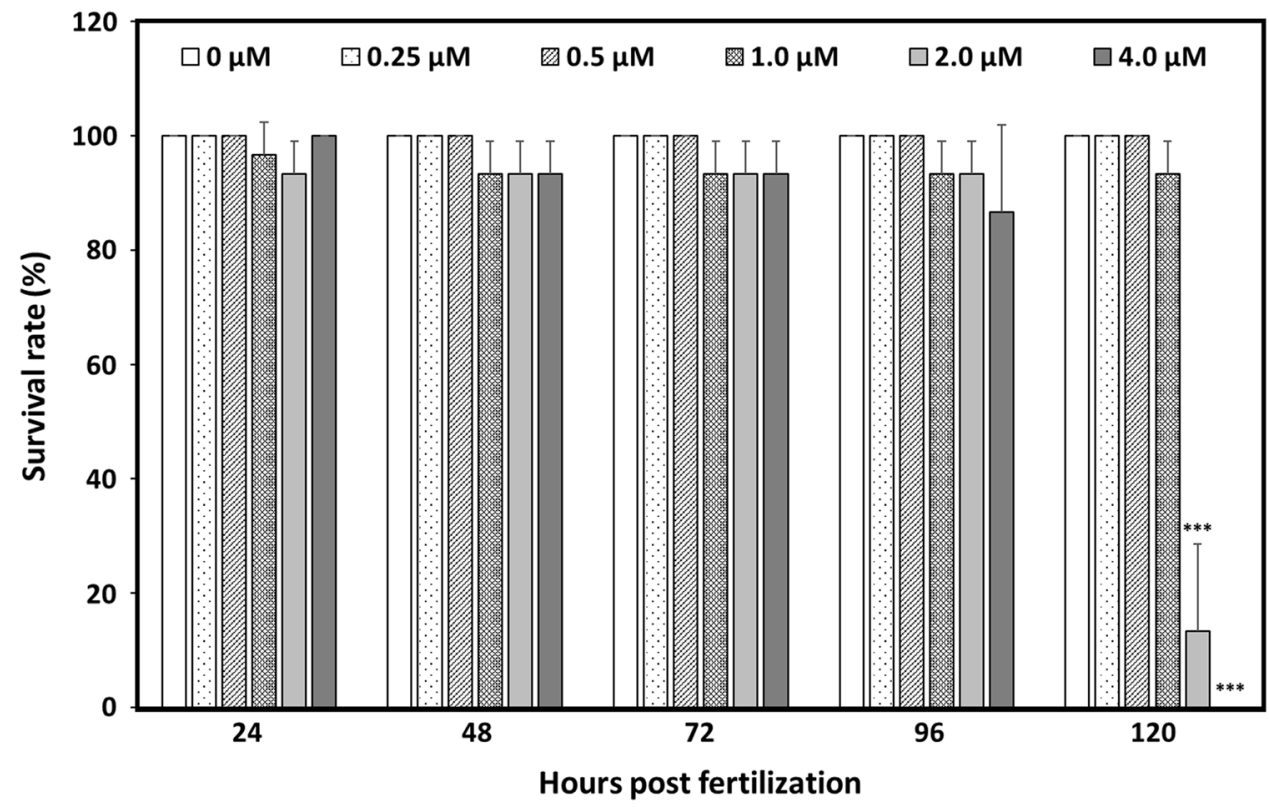

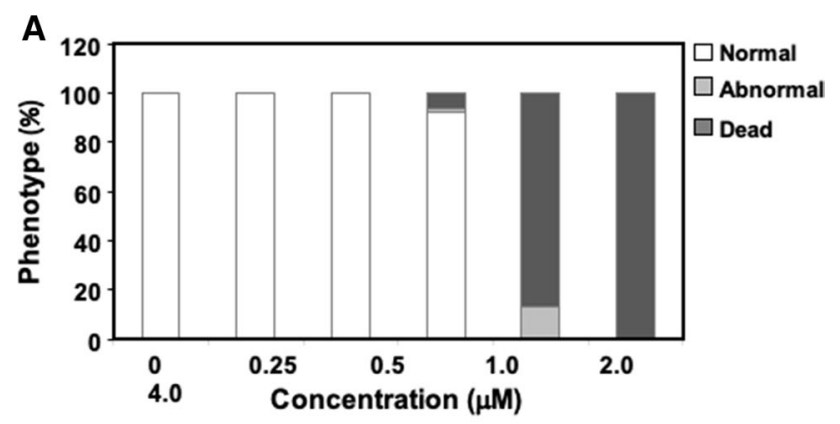

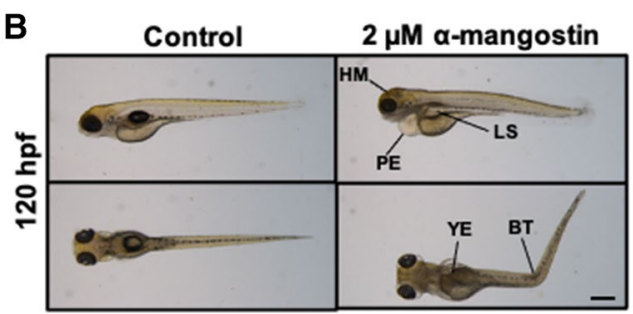

Fig. 2 a Percentages of phenotypes of zebrafish larvae at $120 \mathrm{hpf}$ after treatment with $0.25,0.5,1,2$, and $4 \mu \mathrm{M}$ of $\alpha$-mangostin. The data analysis was performed using data from three independent experiment. b Morphological features of zebrafish larvae at $120 \mathrm{hpf}$ after exposure to $2 \mu \mathrm{M} \alpha$-mangostin compared with those in the control. $H M$ head malformation, $P E$ pericardial oedema, $L S$ absence of swim bladder, $Y E$ yolk oedema, $B T$ bent tail. Scale bar $500 \mu \mathrm{m}$

\section{Exposure to a-mangostin during hepatogenesis causes mortality and malformation in zebrafish larvae}

To assess the effects of $\alpha$-mangostin during hepatogenesis, we used two times ranges of $\alpha$-mangostin exposure. The first exposure extended from $16 \mathrm{hpf}$, when the hepatocyte precursors appear, to $72 \mathrm{hpf}$, when the formation of blood vessels is essentially complete (Pack et al. 1996; Isogai et al. 2001). The second exposure started at $72 \mathrm{hpf}$ and ended when the liver was fully developed at $120 \mathrm{hpf}$. First, $16 \mathrm{hpf} \operatorname{Tg}$ (fabp10a:DsRed) transgenic zebrafish embryos were treated with $0-8 \mu \mathrm{M}$ of $\alpha$-mangostin until $72 \mathrm{hpf}$. The mortality rates were confirmed during these time ranges to find the lowest observed adverse effect level (LOAEL) during the hepatogenesis. This concentration was excluded in the study of the transcriptional effects to omit the non-specific effect of $\alpha$-mangostin on other organs besides the liver. After the exposure from 16 to 72 hpf, mortality and malformations of zebrafish larvae were observed only in the $8-\mu \mathrm{M} \alpha$-mangostin-treated group (Fig. 3a). At 72 hpf, pericardial oedema, yolk oedema, and curved-axis larvae were observed (Fig. 3b). These results imply that at $8 \mu \mathrm{M}$ the compound was toxic to the cardiovascular system, and also to bone formation of zebrafish embryos. The $\alpha$-mangostin exposure that extended from 72 to 120 hpf caused $100 \%$ mortality of zebrafish larvae at $8 \mu \mathrm{M}$ but no mortality or malformations were observed at lower concentrations (Fig. 3c). The higher mortality in the older embryos at $8 \mu \mathrm{M} \alpha$-mangostin may reflect the possession of a chorion by the younger embryos, which protects them from direct exposure to the compound. After $72 \mathrm{hpf}$, when the larvae have hatched, $\alpha$-mangostin is able to come into direct contact with the larvae. Because we aimed to study specific effects of $\alpha$-mangostin on the liver, we did not include the $8 \mu \mathrm{M}$ treatment group in the subsequent experiments.

\section{Alpha-mangostin alters gene expression during hepatogenesis}

To investigate the effects of $\alpha$-mangostin on inflammation, oxidative damage, apoptosis, and hepatic cell function, 
Fig. 3 Zebrafish larvae after treated with $0.5,1,2,4$, and $8 \mu \mathrm{M}$ of $\alpha$-mangostin during hepatogenesis. a Cumulative survival rate of zebrafish larvae at 24,48 , and $72 \mathrm{hpf}$ during the exposure from 16 up to $72 \mathrm{hpf}$. b Morphological features of zebrafish larvae at $72 \mathrm{hpf}$ after exposed to different concentrations of $\alpha$-mangostin from 16 up to $72 \mathrm{hpf}$. Scale bar $100 \mu \mathrm{m}$. c Cumulative survival rate of zebrafish larvae after $48 \mathrm{~h}$ exposure (72-120 hpf). Data in a and c are presented as means \pm SE. The experiment was performed in triplicate. Significant differences were analysed using one-way ANOVA followed by Tukey's HSD test. $* * * p<0.001$ versus control
A
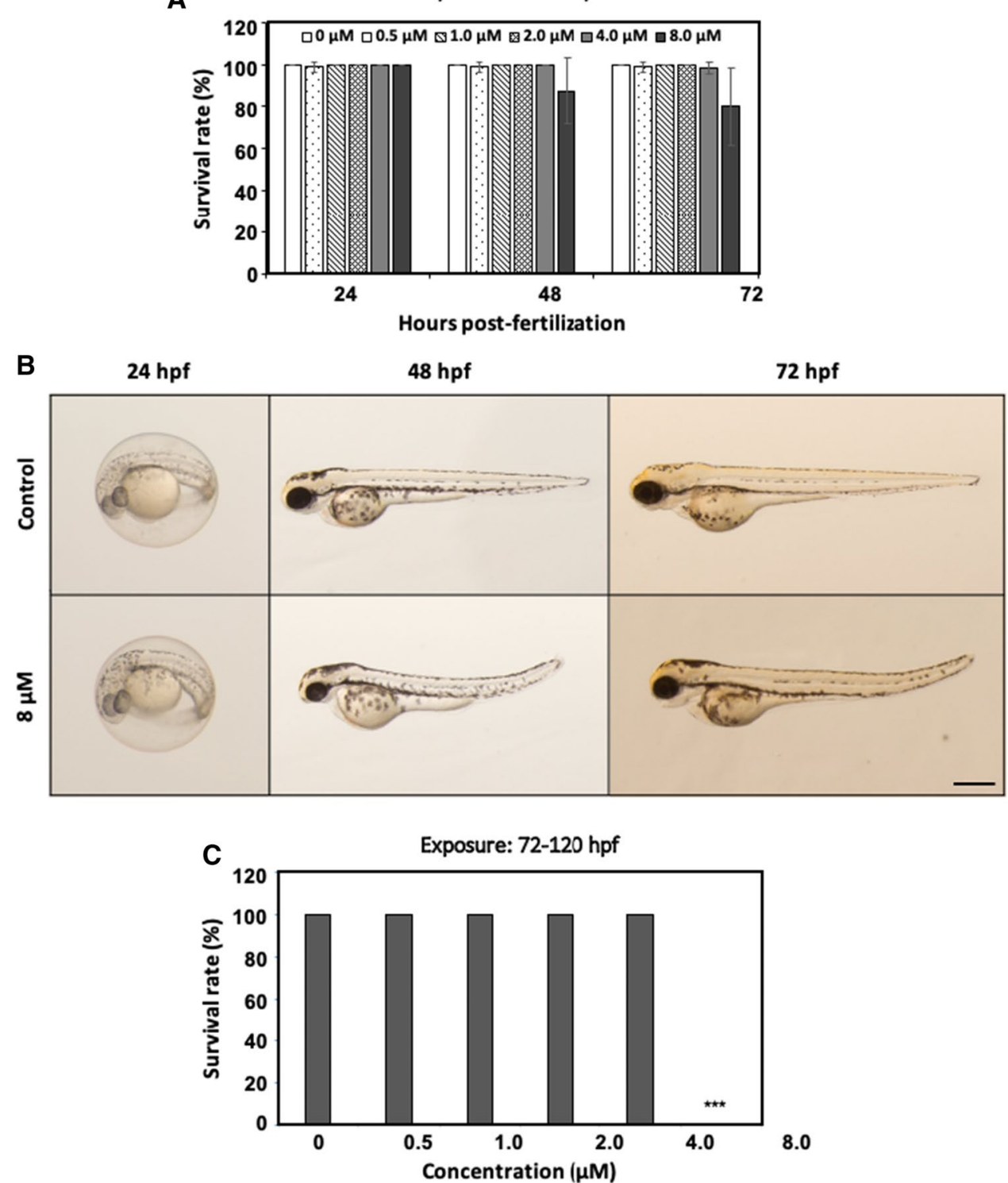

we measured the expression levels of selected genes using qRT-PCR. These genes are listed in Table S1. Transforming growth factor beta (Tgf- $\beta$ ) and tumour necrosis factor alpha (Tnf- $\alpha$ ) are inflammatory markers. Superoxide dismutase (Sod) and catalase (Cat) are markers for oxidative damage. B-cell lymphoma 2 (Bcl-2) and Bcl-2-associated X (Bax) are markers for apoptosis. Caspase- 3 and Caspase- 9 are caspase family members playing essential roles in programmed cell death (including apoptosis, pyroptosis, and necroptosis) and inflammation. Fabp10a is a marker for hepatic cells, and Cyp1a is an enzyme expressed in hepatic cells and involved in phase I xenobiotic and drug metabolisms. The qRT-PCR data for the selected genes is shown in Fig. 4. After 16-72 hpf, $\alpha$-mangostin increased the expression levels of Tgf- $\beta$, Tnf- $\alpha$, and Sod (Fig. 4a) and of Bcl, Bax, Caspase-3, and Caspase-9. The expression of Cypla was increased at $1 \mu \mathrm{M}$ but was not significantly different at $4 \mu \mathrm{M}$. Expression of Fabp10a was reduced in a concentration-dependent manner. These observations imply that $\alpha$-mangostin causes inflammation and oxidative damage, leading to caspase-dependent apoptosis of hepatic cells. Alpha-mangostin-induced caspase-dependent apoptosis via inflammation and oxidative stress was reported in several previous studies using in vitro models. For example, Lee et al. (2017) investigated the effects of $\alpha$-mangostin in cervical cancer cells. The compound increased $\mathrm{Bcl}-2$ and $\mathrm{Bax}$, and also activated Caspase- 3 and Caspase- 9 through reactive oxygen species. In another study, $\alpha$-mangostin induced caspase-mediated apoptosis in human oral squamous cell carcinoma (Kwak et al. 2016). Gene expression levels were also determined after 72-120 hpf, as shown in Fig. 4b. Alpha-mangostin increased the expression of Tgf- $\beta$, Sod, Cat, and Bax. However, the 
Fig. 4 Quantitative RT-PCR analysis of zebrafish larval mRNA expression levels in control and $\alpha$-mangostin-treated groups. Zebrafish embryos were treated with 1 and $4 \mu \mathrm{M}$ $\alpha$-mangostin from: a 16-72 hpf; and b $72-120$ hpf. Expression levels of genes related to L-FABP ( fabp10 and cypla), inflammation (tgf- $b$ and thf$a$ ), oxidative stress ( $\operatorname{sod}$ and $c a t$ ), and apoptosis (bcl, bax, caspase-3, and caspase-9) are shown. The gene expression changes were normalized to $\beta$-actin. The results are presented as mean \pm SE of the relative gene expression level compared with control. Significant differences were calculated using one-way ANOVA followed by Tukey's HSD test. Asterisks indicate significant differences from control group $(* p<0.05 ; * * p<0.01)$
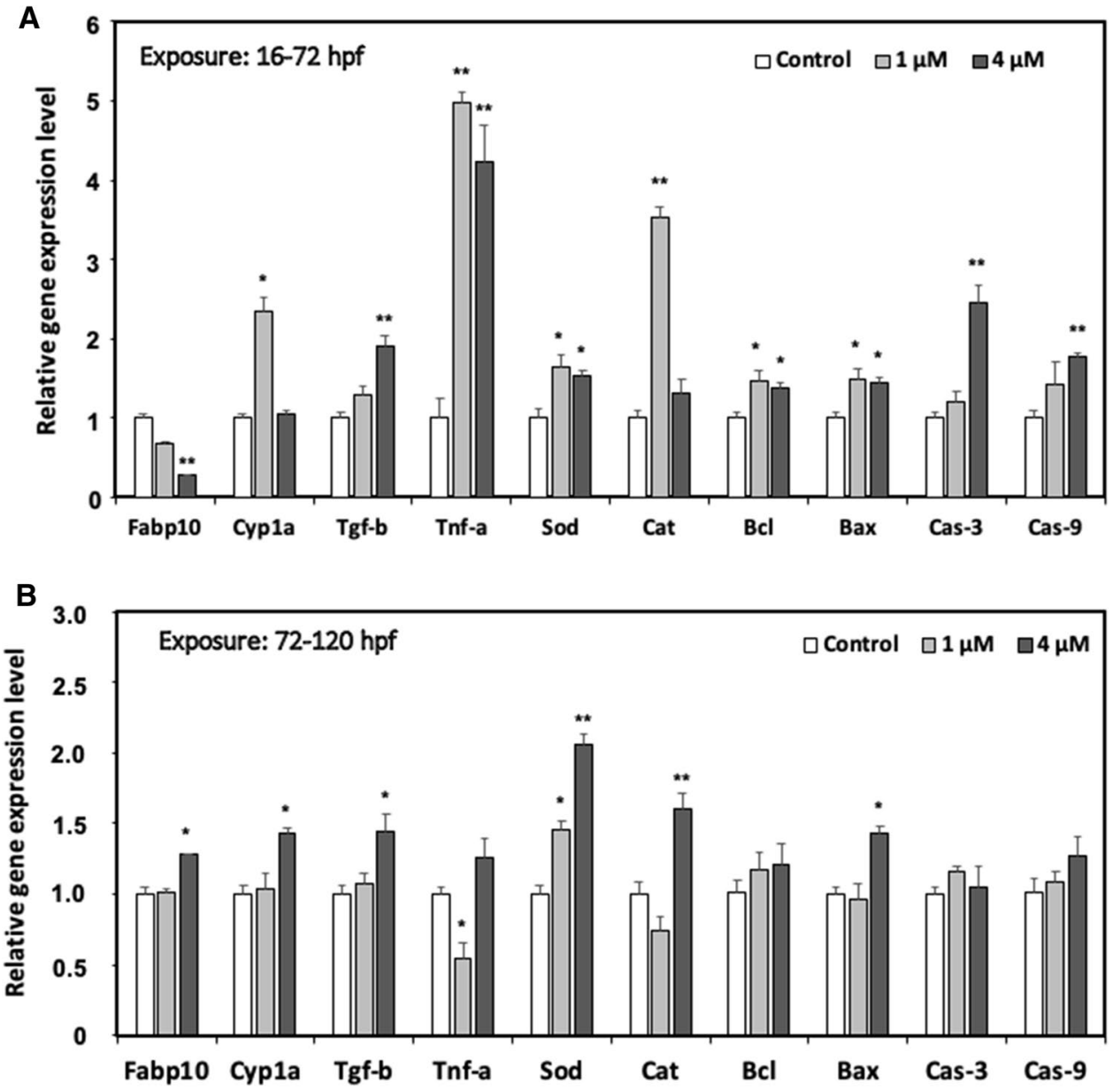

expressions of Bcl, Caspase-3, and Caspase-9 were not significantly altered. We observed an increase of Bax, but no change of $\mathrm{Bcl}$, Caspase- 3 or Caspase-9. This suggests that $\alpha$-mangostin does not fully induce apoptosis, because it induces apoptosis via the caspase family pathway. In addition, at $4 \mu \mathrm{M}$ the compound increased Fabp10a and Cyp1a. These findings indicate that, although larval mortality was sensitive to $\alpha$-mangostin, at this stage (72-120 hpf) larvae were not sensitive to $\alpha$-mangostin-induced apoptosis at the transcriptional level. Moreover, at a concentration of $4 \mu \mathrm{M}$, $\alpha$-mangostin activated expression of Fabp10a and Cyp1a rather than inhibiting hepatic proliferation, as seen in the test in the younger stages. Although survival rates of zebrafish larvae were more sensitive at the later stages (72-120 hpf), the qRT-PCR data indicated that $\alpha$-mangostin had a greater effect on transcription levels before hepatic outgrowth and expansion than during later stages of liver development.

\section{Effects of a-mangostin on liver phenotype}

Transgenic $\operatorname{Tg}$ (Fabp10a:DsRed) zebrafish were used to assess the effects of $\alpha$-mangostin on liver development. These zebrafish exhibit red fluorescent protein (DsRed) specifically in the liver, localizing expression of fatty acid-binding protein 10a (Fabp10a). This protein plays an important role in the intracellular binding and trafficking of long-chain fatty acids in hepatocytes (Her et al. 2003). Fabp10a impairment leads to liver pathogenesis, whereas Fabp10a upregulation protects hepatocytes from oxidative stress (Fan et al. 2013; Smathers et al. 2013). After $\alpha$-mangostin exposure during 16-72 hpf, liver morphology of 72 hpf Tg(fabp10a:DsRed) zebrafish was examined (Fig. 5). At $4 \mu \mathrm{M}$, two populations of larvae were observed. The first population exhibited a small area of fluorescence in the liver (middle panels of Fig. 5); in the second population (lower panels of Fig. 5), the same area was similar to that in the control group (upper panels of Fig. 5). This phenomenon of zebrafish larvae treated with the same concentration of a compound showing different phenotypes was also observed in previous studies. For example, qRTPCR analysis showed that zebrafish drl.3 morphants with either normal or decreased gata1:RFP ${ }^{+}$circulating cells, exhibited comparable levels of drl.3 knockdown (Pimtong et al. 2014). Accordingly, in the present study, $\alpha$-mangostintreated larvae with normal or decreased fabp10a:DsRed ${ }^{+}$ hepatic cells could have similar levels of fabp10a. However, 

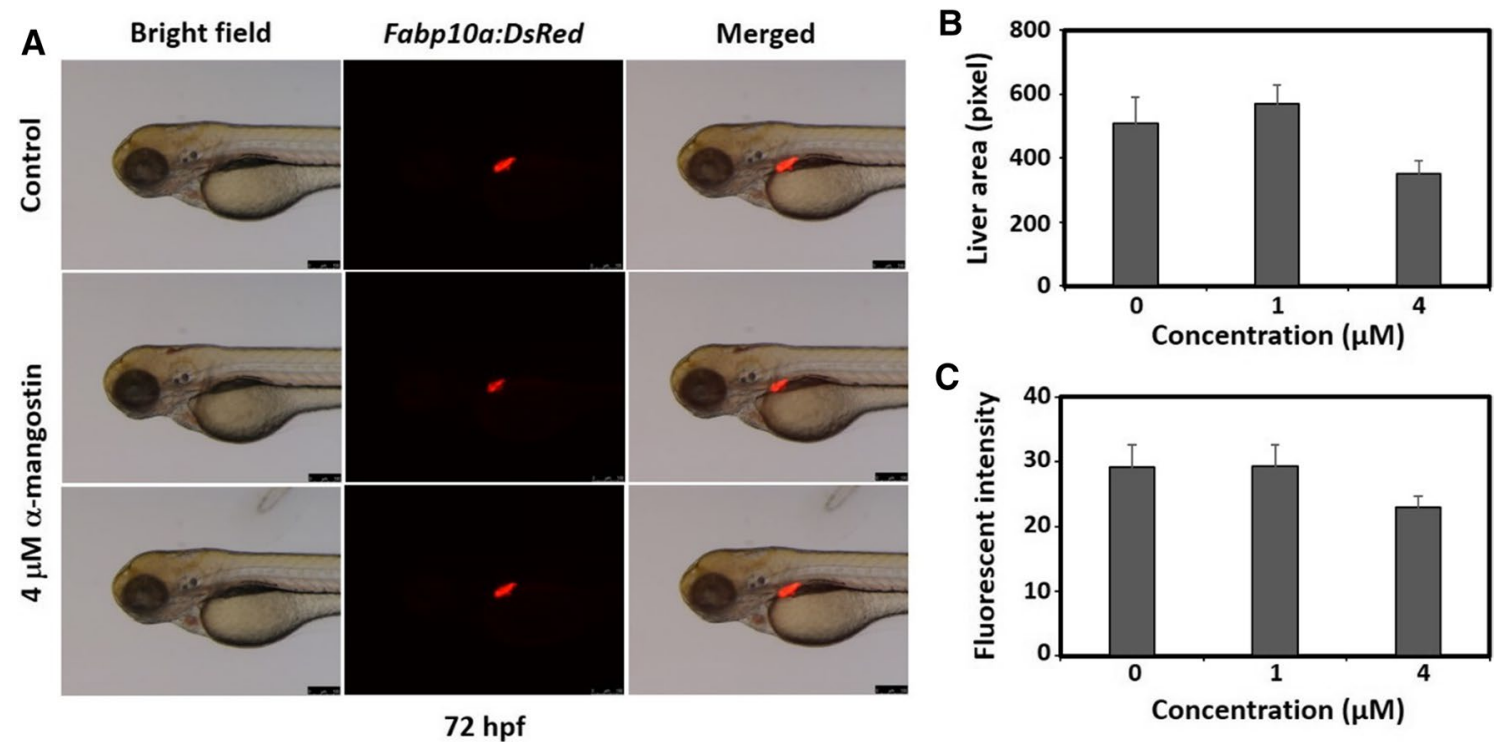

Fig. 5 Effects of $\alpha$-mangostin on hepatogenesis of $\operatorname{Tg}$ (fabp10a:DsRed) zebrafish embryos. Zebrafish embryos were treated with 1 and $4 \mu \mathrm{M} \alpha$-mangostin from 16 to $72 \mathrm{hpf}$. a Representative images of liver morphology in zebrafish larvae at $72 \mathrm{hpf}$ in controls and $\alpha$-mangostin-treated groups. Scale bar $100 \mu \mathrm{m}$. b

whether $\alpha$-mangostin decreases the protein expression level of fabp 10a requires further investigation.

We quantified the intensity and area of fluorescence in zebrafish larvae. At $4 \mu \mathrm{M}$, the average intensity and area of red fluorescence were slightly decreased. However, this phenotypic observation was not statistically significant and was inconsistent with the gene expression results, in which Fabp10a was shown to significantly reduced. $\mathrm{Tg}$ (Fabp10a:DsRed) transgenic zebrafish larvae were also used for the exposure from 72 to $120 \mathrm{hpf}$. After the exposure, the fluorescence area and intensity in the liver of the larvae were not significantly different from those in the control group (Fig. S1). In summary, these results imply that $\alpha$-mangostin shows specific toxicity towards transcriptional levels in early hepatogenesis (16-72 hpf), but not in stages later than $72 \mathrm{hpf}$.

\section{Conclusion}

Alpha-mangostin induced mortality during zebrafish embryonic development. This compound also caused deformities in zebrafish embryos, including head malformation, pericardial oedema, absence of swim bladder, yolk oedema, and bent tail. At the early stage of hepatogenesis (16-72 hpf), $\alpha$-mangostin increased expression levels of genes associated with inflammation, oxidative stress, and caspase-dependent apoptosis, leading to decrease of Fabp10a and Cyp1a. However, significant effects were not
Quantitative analysis of the area of liver fluorescence in control and $\alpha$-mangostin-treated zebrafish larvae. c Quantitative analysis of liver fluorescence intensity in control and $\alpha$-mangostin-treated zebrafish larvae. Data are presented as means \pm SE (15 larvae at each concentration)

detected in liver morphology of transgenic zebrafish larvae. In contrast, $\alpha$-mangostin did not change gene expression associated with caspase-dependent apoptosis during later stages of hepatogenesis at $72-120 \mathrm{hpf}$. Although the concentrations and exposure times of $\alpha$-mangostin used in this study did not induce liver damage, higher concentrations and longer exposure times of the compound might do so. Therefore, it is necessary to be aware of such effects when using $\alpha$-mangostin as a drug. The effects of $\alpha$-mangostin on the fully developed liver require further elucidation.

Acknowledgements This study was supported by the Korea Foundation for Advanced Studies: International Scholar Exchange Fellowship (Funding to W. Pimtong), Republic of Korea.

Author contributions Woo-Keun Kim conceived and designed the experiments. Wittaya Pimtong performed the experiments. Wittaya Pimtong and Hang-Suk Chun analysed the data. Woo-Keun Kim contributed reagents/materials/analytical tools. Wittaya Pimtong, Wannakarn Kitipaspallop and Woo-Keun Kim wrote the paper.

\section{Compliance with ethical standard}

Conflict of interest The authors declare that they have no competing interests.

Ethical approval All experimental protocols and procedures were approved and conducted according to the approved guidelines and regulations of the Institutional Animal Care and Use Committee (IACUC) of the Korea Institute of Toxicology (KIT-1907-0263). 
Open Access This article is licensed under a Creative Commons Attribution 4.0 International License, which permits use, sharing, adaptation, distribution and reproduction in any medium or format, as long as you give appropriate credit to the original author(s) and the source, provide a link to the Creative Commons licence, and indicate if changes were made. The images or other third party material in this article are included in the article's Creative Commons licence, unless indicated otherwise in a credit line to the material. If material is not included in the article's Creative Commons licence and your intended use is not permitted by statutory regulation or exceeds the permitted use, you will need to obtain permission directly from the copyright holder. To view a copy of this licence, visit http://creativecommons.org/licenses/by/4.0/.

\section{References}

Brito LC, Berenger ALR, Figueiredo MR (2017) An overview of anticancer activity of Garcinia and Hypericum. Food Chem Toxicol 109:847-862

Chen G, Li Y, Wang W, Deng L (2018) Bioactivity and pharmacological properties of $\alpha$-mangostin from the mangosteen fruit: a review. Expert Opin Ther Pat 28:415-427

Choi YH, Han SY, Kim YJ et al (2014) Absorption, tissue distribution, tissue metabolism and safety of alpha-mangostin in mangosteen extract using mouse models. Food Chem Toxicol 66:140-146

Duncan SA (2013) Mechanisms controlling early development of the liver. Mech Dev 120:19-33

Fan W, Chen K, Zheng G et al (2013) Role of liver fatty acid binding protein in hepatocellular injury: effect of $\mathrm{CrPic}$ treatment. J Inorg Biochem 124:46-53

Field HA, Ober EA, Roeser T, Stainier DY (2003) Formation of the digestive system in zebrafish. I Liver morphogenesis. Dev Biol 253:279-290

Gavrilas LI, Ionescu C et al (2016) The role of bioactive dietary components in modulating miRNA expression in colorectal cancer. Nutrients 8:E590

Han SY, You BH, Kim YC et al (2015) Dose-independent ADME properties and tentative identification of metabolites of alpha-mangostin from Garcinia mangostana in mice by automated microsampling and UPLC-MS/MS methods. PLoS One 10:e131587

Her GM, Yeh YH, Wu JL (2003) 435-bp liver regulatory sequence in the liver fatty acid binding protein (L-FABP) gene is sufficient to modulate liver regional expression in transgenic zebrafish. Dev Dyn 227:347-356

Hientz K, Mohr A, Bhakta-Guha D, Efferth T (2017) The role of p53 in cancer drug resistance and targeted chemotherapy. Oncotarget 8:8921-8946

Isogai S, Horiguchi M, Weinstein BM (2001) The vascular anatomy of the developing zebrafish: an atlas of embryonic and early larval development. Dev Biol 230:278-301

Ito T, Ando H, Handa H (2011) Teratogenic effects of thalidomide: molecular mechanisms. Cell Mol Life Sci 68:1569-1579

Jindarat S (2014) Xanthones from mangosteen (Garcinia mangostana): multi-targeting pharmacological properties. J Med Assoc Thai 97(2):S196-201

Kim HM, Kim YM et al (2017) Alpha-mangostin ameliorates hepatic steatosis and insulin resistance by inhibition $\mathrm{C}-\mathrm{C}$ chemokine receptor 2. PLoS One 12:e179204
Kittipaspallop W, Taepavarapruk P, Chanchao C, Pimtong W (2019) Acute toxicity and teratogenicity of $\alpha$-mangostin in zebrafish embryos. Exp Biol Med 243:1212-1219

Korzh S, Emelyanov A, Korzh V (2001) Developmental analysis of ceruloplasmin gene and liver formation in zebrafish. Mech Dev 103:137-139

Kumar V, Bhatt PC, Kaithwas G et al (2016) $\alpha$-Mangostin mediated pharmacological modulation of hepatic carbohydrate metabolism in diabetes induced Wistar rat. Beni Seuf Univ J Appl Sci 5:255-276

Kwak HH, Kim ER et al (2016) $\alpha$-Mangostin induces apoptosis and cell cycle arrest in oral squamous cell carcinoma cell. Evid Based Complement Altern Med 2016:5352412. https://doi. org/10.1155/2016/5352412

Lee CH, Ying TH, Chiou HL et al (2017) Alpha-mangostin induces apoptosis through activation of reactive oxygen species and ASK1/p38 signaling pathway in cervical cancer cells. Oncotarget 8:47425-47439

Nelli GB, As K, Kilari EK (2013) Antidiabetic effect of alpha-mangostin and its protective role in sexual dysfunction of streptozotocin induced diabetic male rats. Syst Biol Reprod Med 59:319-328

Ober EA, Field HA, Stainier DY (2003) From endoderm formation to liver and pancreas development in zebrafish. Mech Dev 120:5-18

OECD Test No. 236 (2013) OECD Guidelines for the Testing of Chemicals, Section 2. OECD Publishing, Paris

Pack M, Solnica-KrezelL MJ et al (1996) Mutations affecting development of zebrafish digestive organs. Development 123:321-328

Pedraza-Chaverri J, Cardenas-Rodriguez N, Orozco-Ibarra M, PerezRojas JM (2008) Medicinal properties of mangosteen (Garcinia mangostana). Food Chem Toxicol 46:3227-3239

Pimtong W, Datta M, Ulrich AM, Rhodes J (2014) Drl.3 governs primitive hematopoiesis in zebrafish. Sci Rep 4:5791

Rahmaniah R, Yuyuntia Y (2018) Alpha mangostin inhibits hepatic stellate cells activation through TGF-beta/Smad and Akt signaling pathways: an in vitro study in LX2. Drug Res 68:153-158

Santoriello C, Zon LI (2012) Hooked! Modeling human disease in zebrafish. J Clin Investig 122:2337-2343

Smathers RL, Galligan JJ, Shearn CT et al (2013) Susceptibility of $\mathrm{L}-\mathrm{FABP}^{-1-}$ mice to oxidative stress in early-stage alcoholic liver. J Lipid Res 54:1335-1345

Supawadee S, Thanet S, Wisut P et al (2015) Investigation of therapeutic effects of alpha-mangostin on thioacetamide-induced cirrhosis in rats. J Med Assoc Thai 98(9):S91-97

Vargesson N (2015) Thalidomide-induced teratogenesis: history and mechanisms. Birth Defects Res C. Embryo Today 105:140-156

Wang MH, Zhang KJ, Gu QL et al (2017) Pharmacology of mangostins and their derivatives: a comprehensive review. Chin J Nat Med 15:81-93

Zhang X, Li C, Gong Z et al (2014) Development of a convenient in vivo hepatotoxin assay using a transgenic zebrafish line with liver-specific DsRed express. PLoS One 9:e91874

Zhang KJ, Gu QL, Yang K et al (2017) Anticarcinogenic effects of alpha-mangostin: a review. Planta Med 83:188-202

Publisher's Note Springer Nature remains neutral with regard to jurisdictional claims in published maps and institutional affiliations 